\title{
Dynamically Relevant Local Coordinates for Halo Orbits
}

\author{
Eric D. Gustafson* and Daniel J. Scheeres ${ }^{\dagger}$
}

\begin{abstract}
A local coordinate system based on the eigenstructure of the Halo orbit is proposed. We show that one only needs to keep track of six intuitive scalars to easily understand the qualitative dynamic evolution of spacecraft states near a halo orbit. Special attention is given to the center subspace and the space associated with the unity eigenvalues of the monodromy matrix. Examples are given for halo orbits in the Hill Three-Body Problem.
\end{abstract}

\section{Introduction}

Halo orbits have shown great usefulness in space missions. The Wilkinson Microwave Anisotropty Probe (WMAP), near the Earth-Sun $L_{2}$ point is studying the cosmic microwave background radiation and the origins of the universe while the Solar and Heliospheric Observatory (SOHO) is currently studying the Sun from an orbit about $L_{1}$. Dynamical Systems Theory, when applied to halo orbits, has allowed missions such as Genesis to use very little fuel while still accomplishing complicated objectives. Our goal is to make the analysis of trajectories near halo orbits more intuitive.

When one numerically integrates a halo orbit, the simulation is typically carried out in a Lagrangian or Hamiltonian coordinate frame. When studying trajectories close to the periodic orbit, the dynamics may be linearized using the same frame in which the full nonlinear simulation was computed. This frame does not provide clear insight on how the spacecraft moves when it is in close proximity to a halo orbit. We propose a more dynamically intuitive local coordinate system based on the eigenstructure of the linearization. This method provides a simple way to compute and understand spacecraft motion near a halo orbit in terms of the local dynamical manifolds.

The monodromy matrix associated with a particular halo orbit is defined as the state transition matrix (STM) evaluated after one period of the orbit $(T)$, denoted by $\Phi(T, 0)$. The interpretation of $\Phi(T, 0)$ is that it maps the local state forward by one orbit period. Therefore, the eigenstructure of $\Phi(T, 0)$ describes how spacecrafts behave near the nominal halo orbit. For all Hamiltonian systems, the STM is symplectic, and in the cases we study, $\Phi(T, 0)$ has the following six eigenvalues:

- Stable real eigenvalue, $\lambda_{s},\left|\lambda_{s}\right|<1$

- Unstable real eigenvalue, $\lambda_{u},\left|\lambda_{u}\right|>1$

- Two neutrally stable eigenvalues, $\lambda_{ \pm}=e^{ \pm i \theta},\left|\lambda_{ \pm}\right|=1$

- Two unity eigenvalues with algebraic multiplicity two, $\lambda_{1}=1$

The underlying Hamiltonian nature of the system allows us to analytically derive a left and right eigenvector of $\Phi(T, 0)$. For any small deviation in the direction of the dynamic flow, the state will be mapped to itself after one period. In other words, the direction of the flow is a right eigenvector of $\Phi(T, 0)$ associated with the unity eigenvalue. Additionally, when we combine the knowledge that these linear deviations do not capture changes in energy and periodic orbits of a given energy are isolated in phase space for a Hamiltonian system, ${ }^{1,2}$ we can determine that this is the only available eigenvector for the unity eigenvalue. In other words, the unity eigenvalue has algebraic multiplicity two and geometric multiplicity one. Therefore, the monodromy matrix is defective, i.e., there is not a full linearly independent basis of eigenvectors which we can use to decompose the state. The direction that is not spanned by the five available eigenvectors is the gradient of the energy with respect to the state; $\frac{d H}{d X}$. This defectiveness is also present in other simple astrodynamic problems such as the planar restricted two-body problem. Also, it is easy to show that $\left(\frac{d H}{d X}\right)^{\mathrm{T}}$ is a left-eigenvector of the monodromy matrix.

${ }^{*}$ Ph. D. Candidate, Department of Aerospace Engineering, University of Michigan, 1320 Beal Ave, Ann Arbor, MI 48109-2140, email: edgus@umich.edu

${ }^{\dagger}$ A. Richard Seebass Professor, Aerospace Engineering Sciences, Colorado Center for Astrodynamics Research, University of Colorado, Engineering Center, ECOT 611, 429 UCB, Boulder, CO 80309-0429, email: scheeres@ colorado.edu, Member AAS, Associate Fellow AIAA 
We wish to choose coordinates to describe the state which convey dynamical meaning. Instead of carrying over the coordinate basis from the linearization of the Lagrangian or Hamiltonian coordinate frame, we will make use the linear manifolds, surface of section, and energy concepts. The energy component is the only component that cannot be dealt with using the available eigenvectors of the monodromy matrix. The six coordinates necessary to fully quantify the state, which be described in more detail shortly, are

1. component along the unstable manifold, $a_{u}$

2. component along the stable manifold, $a_{s}$

3. pseudo-magnitude of state projected into the center subspace, $\rho$

4. pseudo-angle of the state projected into the center subspace, $\gamma$

5. deviation from the surface of section (downtrack deviation), $a_{d}$

6. deviation in energy, $\delta H$

The unstable and stable components are straightforward to calculate by taking the dot product of the left eigenvector of $\Phi(T, 0)$ with the local state. The center components, and the components associated with the unity eigenvalues merit more attention, however.

For the center manifold, we propose the use of pseudo-magnitude and pseudo-angle components, $\rho$ and $\gamma$. This is to give more clear insight as to how the coordinates change with time. For example, with no control acting, $\gamma$ will be nearly constant throughout the motion and $\rho$ will be periodic with the same period as the halo orbit.

The downtrack deviation, $a_{d}$, may be computed by a simple dot product in the same way as the hyperbolic manifolds. The remaining component may be taken as either the energy deviation or the generalized left-eigenvector dot product. Each has its own advantages and disadvantages. The generalized eigenvector arguably gives a more elegant computation, however, if a primary interest of the analysis is the deviation in energy, then it may be more useful to simply use it as a coordinate.

Halo orbits and the STM for the examples in this paper were integrated using an 8th order accurate, arbitrary precision, symplectic Runge-Kutta integration scheme with precision set to 256 bits. ${ }^{3}$ Initial conditions for the halo orbits were found by the method developed by Howell. ${ }^{4}$ Throughout this paper, we assume the state and STM are expressed in the Hamiltonian frame unless noted otherwise.

\section{Definitions}

\section{A. Hamiltonian Systems}

A Hamiltonian system is one whose motion is determined by a scalar function $H(\mathbf{q}, \mathbf{p}, t)$, where the generalized coordinates are $\mathbf{q}$, the generalized momenta are $\mathbf{p}$, and $t$ is time. Both $\mathbf{q}$ and $\mathbf{p}$ are elements of $\mathbb{R}^{n}$. In this analysis, we will assume a time-invariant Hamiltonian as that is representative of many astrodynamic systems. The equations of motion are

$$
\begin{aligned}
& \dot{\mathbf{q}}=\frac{\partial H}{\partial \mathbf{p}} \\
& \dot{\mathbf{p}}=-\frac{\partial H}{\partial \mathbf{q}} .
\end{aligned}
$$

Defining the matrix $J$ as

$$
J=\left[\begin{array}{cc}
0_{n \times n} & I_{n} \\
-I_{n} & 0_{n \times n}
\end{array}\right]
$$

and concatenating the states into $\mathbf{X}=\left[\begin{array}{ll}\mathbf{q}^{\mathrm{T}} & \mathbf{p}^{\mathrm{T}}\end{array}\right]^{\mathrm{T}} \in \mathbb{R}^{2 n}$, we have an alternative description of the equations of motion:

$$
\dot{\mathbf{X}}=J \frac{\partial H}{\partial \mathbf{X}}
$$

Any solution to Eq. (3), $\mathbf{X}\left(t ; \mathbf{X}_{0}\right)$, is said to be periodic if for some $T>0, \mathbf{x}(t+T)=\mathbf{X}(t)$ for all times $t$. 
Hamiltonian and Lagrangian systems are, of course, closely related. Let the Lagrangian coordinates be $\mathbf{q}$ and $\dot{\mathbf{q}}$; the generalized coordinates and generalized velocities. Then our Hamiltonian coordinates will be $\mathbf{q}$ and $\mathbf{p} \equiv \dot{\mathbf{q}}+\omega \times \mathbf{q}$. The vector $\omega$ is the angular velocity between the two frames. The collection of the states can be called $\mathbf{X}_{L}$ for the Lagrangian frame, where $\mathbf{X}_{L}=\left[\begin{array}{ll}\mathbf{q}^{\mathrm{T}} & \dot{\mathbf{q}}^{\mathrm{T}}\end{array}\right]^{\mathrm{T}}$.

Writing this relation in a more compact form, we have

$$
\begin{aligned}
\mathbf{X} & =\left[\begin{array}{cc}
I_{3} & 0_{3 \times 3} \\
\widetilde{\omega} & I_{3}
\end{array}\right] \mathbf{X}_{L} \\
& =T_{\omega} \mathbf{X}_{L} .
\end{aligned}
$$

Also from this we have that $\Phi(T, 0)=T_{\omega} \Phi_{L}(T, 0) T_{-\omega}$ and $\frac{d H}{d X_{L}}=T_{\omega}^{\mathrm{T}} \frac{d H}{d X}$.

\section{B. Eigenvalues and Eigenvectors}

In this section we define the left and right eigenvectors for a matrix $A$. It is assumed that the dimensions of $A$ are $n \times n$, and that $A$ has a full set of $n$ independent eigenvectors (left or right).

A right-eigenvector (a column vector), $\mathbf{v}_{i}^{R}$, and its associated eigenvalue, $\lambda_{i}$, are defined to satisfy the following relation:

$$
A \mathbf{v}_{i}^{R}=\lambda_{i} \mathbf{v}_{i}^{R}
$$

Similarly, a left-eigenvector (a row vector), $\mathbf{v}_{i}^{L}$, satisfies

$$
\mathbf{v}_{i}^{L} A=\lambda_{i} \mathbf{v}_{i}^{L}
$$

Eqs. (4) and (5) hold for each of the $n$ eigenvalues of the $A$, however, a more compact notion may be achieved by placing the right-eigenvectors into the columns of matrix $R$ and the left-eigenvectors into the rows of matrix $L$ such that the $i$ th column of $R$ is $\mathbf{v}_{i}^{R}$ and the $i$ th row of $L$ is $\mathbf{v}_{i}^{L}$ :

$$
\begin{gathered}
R=\left[\begin{array}{llllll}
\mathbf{v}_{1}^{R} & \mid & \mathbf{v}_{2}^{R} & \mid & \cdots & \mid \\
\mathbf{v}_{n}^{R}
\end{array}\right] \\
L=\left[\begin{array}{c}
\mathbf{v}_{1}^{L} \\
- \\
\mathbf{v}_{2}^{L} \\
- \\
\vdots \\
- \\
\mathbf{v}_{n}^{L}
\end{array}\right]
\end{gathered}
$$

Additionally, define the matrix $D$ to be a matrix with the eigenvalues on the diagonal and zeros elsewhere:

$$
D_{i j}= \begin{cases}\lambda_{i}, & i=j \\ 0, & i \neq j\end{cases}
$$

Now, Eqs. (4) and (5) may be written as

$$
A R=R D
$$

and

$$
L A=D L
$$

Premultiplying and postmultiplying Eq. (9) by $R^{-1}$ yields $\left(R^{-1}\right) A=D\left(R^{-1}\right)$, which is of the same form as Eq. (10) and therefore $L$ can be defined to be $R^{-1}$, which gives us the left-eigenvectors from the right-eigenvectors. Also, note that $A$ may be decomposed as

$$
\begin{aligned}
A & =R D L \\
& =\lambda_{1} \mathbf{v}_{1}^{R} \mathbf{v}_{1}^{L}+\cdots+\lambda_{n} \mathbf{v}_{n}^{R} \mathbf{v}_{n}^{L}
\end{aligned}
$$




\section{Symplectic Matrices}

A symplectic matrix, $M$, is one which satisfies

$$
M^{\mathrm{T}} J M=J .
$$

Symplectic matrices have many useful properties, one of which is that given an eigenvalue, $\lambda$, and corresponding right eigenvector, $\mathbf{v}$, then $\lambda^{-1}$ is also an eigenvalue and corresponds to the left eigenvector $(J \mathbf{v})^{\mathrm{T}}$. Also worth noting is that if $M$ is real, then the eigenvalues occur in complex conjugate pairs, so that if $\lambda$ is complex and $|\lambda| \neq 1$ with eigenvector $\mathbf{v}$, then $\lambda^{-1}, \bar{\lambda}$, and $\bar{\lambda}^{-1}$ are all eigenvalues corresponding to left eigenvector $(J \mathbf{v})^{\mathrm{T}}$, right eigenvector $\overline{\mathbf{v}}$, and left eigenvector $(J \overline{\mathbf{v}})^{\mathrm{T}}$, respectively.

Another useful property is ease of inversion: the inverse of a symplectic matrix is simply

$$
M^{-1}=-J M^{\mathrm{T}} J
$$

which follows immediately from Eq. (12).

\section{Properties of Left \& Right Eigenvectors}

\section{A. Dot Products}

When $L$ is defined as $R^{-1}, L$ forms a dual basis, and the dot products between the left and right-eigenvectors have the special property that

$$
\mathbf{v}_{i}^{L} \cdot \mathbf{v}_{j}^{R}=\delta_{i j}
$$

where $\delta_{i j}$ is the Kronecker delta. This property can be derived by considering the multiplication of $L$ and $R$ :

$$
\begin{aligned}
L R & =\left[\begin{array}{c}
\mathbf{v}_{1}^{L} \\
- \\
\vdots \\
- \\
\mathbf{v}_{n}^{L}
\end{array}\right]\left[\begin{array}{lllll}
\mathbf{v}_{1}^{R} & \mid & \cdots & \mid & \mathbf{v}_{n}^{R}
\end{array}\right] \\
& =\left[\begin{array}{cccc}
\mathbf{v}_{1}^{L} \mathbf{v}_{1}^{R} & \mathbf{v}_{1}^{L} \mathbf{v}_{2}^{R} & & \mathbf{v}_{1}^{L} \mathbf{v}_{n}^{R} \\
\mathbf{v}_{2}^{L} \mathbf{v}_{1}^{R} & \mathbf{v}_{2}^{L} \mathbf{v}_{2}^{R} & \ldots & \\
& \vdots & \ddots & \\
\mathbf{v}_{n}^{L} \mathbf{v}_{1}^{R} & & & \mathbf{v}_{n}^{L} \mathbf{v}_{n}^{R}
\end{array}\right] \\
& =\left[\begin{array}{llll}
\mathbf{v}_{1}^{L} \cdot \mathbf{v}_{1}^{R} & \mathbf{v}_{1}^{L} \cdot \mathbf{v}_{2}^{R} & & \mathbf{v}_{1}^{L} \cdot \mathbf{v}_{n}^{R} \\
\mathbf{v}_{2}^{L} \cdot \mathbf{v}_{1}^{R} & \mathbf{v}_{2}^{L} \cdot \mathbf{v}_{2}^{R} & \ldots & \\
& \vdots & \ddots & \\
\mathbf{v}_{n}^{L} \cdot \mathbf{v}_{1}^{R} & & & \mathbf{v}_{n}^{L} \cdot \mathbf{v}_{n}^{R}
\end{array}\right]
\end{aligned}
$$

or

$$
(L R)_{i j}=\mathbf{v}_{i}^{L} \cdot \mathbf{v}_{j}^{R}
$$

Since $L R=I$, this implies that $\mathbf{v}_{i}^{L} \cdot \mathbf{v}_{j}^{R}=\delta_{i j}$

\section{B. Projections of Dynamic State onto Left-Eigenvectors}

\section{Continuous Time Systems}

For a continuous-time, linear, time-varying system, $\dot{\mathbf{x}}=A(t) \mathbf{x}$ with state transition matrix $\Phi(t, 0)$, we have

$$
\mathbf{x}(t)=\Phi(t, 0) \mathbf{x}_{0}
$$

Define the time-varying eigenvalues and left-eigenvectors of the state transition matrix as $\mu_{i}(t)$ and $\mathbf{v}_{i}^{L}(t)$, respectively. Dotting the left-eigenvectors with the previous equation gives

$$
\begin{aligned}
\mathbf{v}_{i}^{L}(t) \mathbf{x}(t) & =\mathbf{v}_{i}^{L}(t) \Phi(t, 0) \mathbf{x}_{0} \\
& =\mu_{i}(t) \mathbf{v}_{i}^{L}(t) \mathbf{x}_{0}
\end{aligned}
$$


Therefore, the time history of a left-eigenvector dotted with the state is simply the left-eigenvector dotted with the initial state, multiplied by the eigenvalue.

When this is applied to a linear time-invariant system, further simplifications can be made. First, note that the state transition matrix is equal to the matrix exponential of $A t$,

$$
\Phi(t, 0)=e^{A t}
$$

Second, note that the eigenvectors of $e^{A t}$ can be taken to be the same as the eigenvectors of $A$ and the eigenvalues of $e^{A t}$ are simply $\mu_{i}(t)=e^{\lambda_{i} t}$, where $\lambda_{i}$ are defined as the eigenvalues of $A$. This gives a useful simplification of the time-varying result:

$$
\begin{aligned}
\mathbf{v}_{i}^{L} \mathbf{x}(t) & =\mu_{i}(t) \mathbf{v}_{i}^{L} \mathbf{x}_{0} \\
& =e^{\lambda_{i} t} \mathbf{v}_{i}^{L} \mathbf{x}_{0}
\end{aligned}
$$

\section{2. $\quad$ Discrete Time Systems}

As could be expected, similar results exists for a discrete-time system. For a discrete-time, linear, time-varying system, $\mathbf{x}(k+1)=A(k) \mathbf{x}(k)$ with state transition matrix $\Psi(k, 0)=A(k-1) \cdots A(0)$, we have

$$
\mathbf{x}(k)=\Psi(k, 0) \mathbf{x}(0)
$$

Define the time-varying eigenvalues and left-eigenvectors of the state transition matrix as $\mu_{i}(k)$ and $\mathbf{v}_{i}^{L}(k)$, respectively. Dotting the left-eigenvectors with the previous equation gives

$$
\begin{aligned}
\mathbf{v}_{i}^{L}(k) \mathbf{x}(k) & =\mathbf{v}_{i}^{L}(k) \Psi(k, 0) \mathbf{x}(0) \\
& =\mu_{i}(k) \mathbf{v}_{i}^{L}(k) \mathbf{x}_{0}
\end{aligned}
$$

As in the continuous-time case, simplifications can be made when the system is time-invariant. First, note that the state transition matrix is equal to the $A$ matrix to a power,

$$
\Psi(k, 0)=A^{k}
$$

Just as in the continuous-time case, the eigenvectors of the state transition matrix are the same as the $A$ matrix. Also, the eigenvalues of $A^{k}$ are simply $\mu_{i}(k)=\lambda_{i}^{k}$, where $\lambda_{i}$ are defined as the eigenvalues of $A$. These results give the the simplified relation that

$$
\begin{aligned}
\mathbf{v}_{i}^{L} \mathbf{x}(k) & =\mu_{i}(k) \mathbf{v}_{i}^{L} \mathbf{x}_{0} \\
& =\lambda_{i}^{k} \mathbf{v}_{i}^{L} \mathbf{x}_{0}
\end{aligned}
$$

\section{Floquet Theory}

Let us consider a time-varying linear system $\dot{\mathbf{x}}=A(t) \mathbf{x}$, with $A(t)$ being periodic with period $T$, i.e. $A(t+T)=$ $A(t)$. An abbreviated statement of Floquet theory states that for any fundamental solution matrix, $\Psi(t)$, there exists a nonsingular matrix $Q$ such that $\Psi(t+T)=\Psi(t) Q$. For a more detailed description of Floquet theory, see Chen, page $153 .{ }^{5}$ If we apply Floquet theory to the state transition matrix $\Phi\left(t_{2}, t_{1}\right)$, which is a fundamental solution matrix satisfying $\Phi\left(t_{1}, t_{1}\right)=I$, we have

$$
\Phi(t+T, 0)=\Phi(t, 0) \Phi(T, 0)
$$

The state transition matrix spanning a length of time equal to one period of the dynamics, $\Phi(T, 0)$, is known as the monodromy matrix. We may recursively apply Eq. (14) to derive other useful identities, such as

$$
\begin{gathered}
\Phi(t+2 T, 0)=\Phi(t+T, 0) \Phi(T, 0) \\
=\Phi(t, 0) \Phi^{2}(T, 0) \\
\vdots \\
\Phi(t+n T, 0)=\Phi(t, 0) \Phi^{n}(T, 0)
\end{gathered}
$$


In general, we can write the state transition matrix between any two times as a function only of $\Phi(t, 0), t \in[0, T]$ (including the monodromy matrix):

$$
\Phi\left(t_{2}+n T, t_{1}+m T\right)=\Phi\left(t_{2}, 0\right) \Phi^{n-m}(T, 0) \Phi^{-1}\left(t_{1}, 0\right)
$$

An example particularly suited to our analysis is the state transition matrix which maps a state at a given time, $t$, to a time that is one period in the future, $t+T$ :

$$
\Phi(t+T, t)=\Phi(t, 0) \Phi(T, 0) \Phi^{-1}(t, 0)
$$

\section{Monodromy Matrix Manifold Projections}

Suppose $\Phi(T, 0)$ is defined as the monodromy matrix for a periodic 3-D halo orbit. Let us consider a monodromy matrix with the following six eigenvalues.

- Stable real eigenvalue, $\lambda_{s},\left|\lambda_{s}\right|<1$

- Unstable real eigenvalue, $\lambda_{u}=\lambda_{s}^{-1},\left(\left|\lambda_{u}\right|>1\right)$

- Two neutrally stable eigenvalues, $\lambda_{ \pm}=e^{ \pm i \theta},\left|\lambda_{ \pm}\right|=1$

- Two unity eigenvalues with algebraic multiplicity two, $\lambda_{1}=1$

\section{A. Jacobi Integral Eigenvectors}

We can derive a left and right eigenvector of $\Phi(T, 0)$ from Hamiltonian dynamics. The direction of the dynamic flow is $J \frac{d H}{d X}$, and any small deviation in that direction will map to itself after one period. That is,

$$
J \frac{d H}{d X}=\Phi(T, 0) J \frac{d H}{d X}
$$

or that $J \frac{d H}{d X}$ is a right eigenvector of $\Phi(T, 0)$ associated with the unity eigenvalue. Additionally, when we combine the knowledge that these linear deviations do not capture changes in energy and periodic orbits of a given energy are isolated in phase space for a Hamiltonian system ${ }^{1,2}$ (with the exception of certain bifurcation points), we can determine that this is the only available eigenvector for the unity eigenvalue. In other words, the unity eigenvalue has algebraic multiplicity two and geometric multiplicity one and $\Phi(T, 0)$ is defective. Therefore, there is not a full linearly independent basis of eigenvectors which we can use to decompose the state. The direction that is not spanned by the five available eigenvectors is the gradient of the energy with respect to the state; $\frac{d H}{d X}$. We will use this in the next section. This is because $\left(\frac{d H}{d X}\right)^{\mathrm{T}}$ is a left-eigenvector of the monodromy matrix as discussed in section II-C.

These eigenvectors may also be derived for the Lagrangian frame by appropriate transformations. In our case, the angular velocity between the frames is $\omega=\left[\begin{array}{lll}0 & 0 & 1\end{array}\right]^{\mathrm{T}}$. Starting with the knowledge that $J \frac{d H}{d X}$ is a right eigenvector for the Hamiltonian system (Eq. (17)), we can convert to the Lagrangian frame to find that

$$
\begin{aligned}
& T_{\omega} \Phi_{L}(T, 0) T_{-\omega} J T_{-\omega}^{\mathrm{T}} \frac{d H}{d X_{L}}=J T_{-\omega}^{\mathrm{T}} \frac{d H}{d X_{L}} \\
& \Phi_{L}(T, 0)\left[T_{-\omega} J T_{-\omega}^{\mathrm{T}} \frac{d H}{d X_{L}}\right]=\left[T_{-\omega} J T_{-\omega}^{\mathrm{T}} \frac{d H}{d X_{L}}\right]
\end{aligned}
$$

or that $\left[T_{-\omega} J T_{-\omega}^{\mathrm{T}} \frac{d H}{d X_{L}}\right]$ is a right-eigenvector for the Lagrangian system. Note that

$$
T_{-\omega} J T_{-\omega}^{\mathrm{T}}=\left[\begin{array}{cc}
0_{3 \times 3} & I_{3}+\widetilde{\omega} \\
-I_{3} & 2 \widetilde{\omega}
\end{array}\right]
$$

Similarly, starting with the left eigenvector of the Hamiltonian system, $\left[\frac{d H}{d X}\right]^{\mathrm{T}}$, we have

$$
\begin{aligned}
{\left[T_{-\omega}^{\mathrm{T}} \frac{d H}{d X_{L}}\right]^{\mathrm{T}}\left[T_{\omega} \Phi_{L}(T, 0) T_{-\omega}\right] } & =\left[T_{-\omega}^{\mathrm{T}} \frac{d H}{d X_{L}}\right]^{\mathrm{T}} \\
{\left[\frac{d H}{d X_{L}}\right]^{\mathrm{T}} \Phi_{L}(T, 0) } & =\left[\frac{d H}{d X_{L}}\right]^{\mathrm{T}}
\end{aligned}
$$


Therefore, $\left[\frac{d H}{d X_{L}}\right]^{\mathrm{T}}$ is a left-eigenvector of the Lagrangian system.

\section{B. Complex Eigenvectors}

Define the right-eigenvectors associated with these eigenvalues as $\mathbf{v}_{s}^{R}, \mathbf{v}_{u}^{R}, \mathbf{v}_{ \pm}{ }^{R}$. The left-eigenvectors, $\mathbf{v}_{s}^{L}, \mathbf{v}_{u}^{L}, \mathbf{v}_{ \pm}{ }^{L}$, are defined as before. Decomposing the monodromy matrix as in Eq. (11) gives

$$
\Phi(T, 0)=\lambda_{s} \mathbf{v}_{s}^{R} \mathbf{v}_{s}^{L}+\lambda_{u} \mathbf{v}_{u}^{R} \mathbf{v}_{u}^{L}+\lambda_{+} \mathbf{v}_{+}^{R} \mathbf{v}_{+}^{L}+\lambda_{-} \mathbf{v}_{-}^{R} \mathbf{v}_{-}^{L}
$$

This form is not particularly useful for physical insight since the eigenvalues and eigenvectors associated with the center manifold are complex. However, we may rearrange to obtain purely real quantities for the eigenvectors.

Instead of using the complex eigenvectors associated with $\lambda_{ \pm}$, it is useful to decompose them into two real vectors $\alpha^{R}$ and $\beta^{R}$. Assuming the eigenvectors associated with $\lambda_{ \pm}$are $\mathbf{v}_{ \pm}^{R}$, we can partition the eigenvectors into real and imaginary parts as follows.:

$$
\begin{aligned}
& \mathbf{v}_{ \pm}^{R}=\frac{1}{\sqrt{2}}\left(\alpha^{R}( \pm)^{r} i \beta^{R}\right) \\
& \mathbf{v}_{ \pm}^{L}=\frac{1}{\sqrt{2}}\left(\alpha^{L}(\mp)^{\ell} i \beta^{L}\right)
\end{aligned}
$$

From this definition, we can derive identities that $\alpha$ and $\beta$ must satisfy by taking the dot product of righteigenvectors with the left-eigenvectors:

$$
\mathbf{v}_{ \pm}^{L} \cdot \mathbf{v}_{ \pm}^{R}=\frac{1}{2}\left(\alpha^{L} \cdot \alpha^{R}( \pm)^{r} i \alpha^{L} \cdot \beta^{R}(\mp)^{\ell} i \alpha^{R} \cdot \beta^{L}-(\mp)^{\ell}( \pm)^{r} \beta^{L} \cdot \beta^{R}\right)
$$

Dotting $\mathbf{v}_{+}^{L}$ with $\mathbf{v}_{+}^{R}$, we have

$$
\frac{1}{2}\left(\alpha^{L} \cdot \alpha^{R}+\beta^{L} \cdot \beta^{R}+i\left(\alpha^{L} \cdot \beta^{R}-\alpha^{R} \cdot \beta^{L}\right)\right)=1
$$

Dotting $\mathbf{v}_{-}^{L}$ with $\mathbf{v}_{+}^{R}$ gives

$$
\frac{1}{2}\left(\alpha^{L} \cdot \alpha^{R}-\beta^{L} \cdot \beta^{R}+i\left(\alpha^{R} \cdot \beta^{L}+\alpha^{L} \cdot \beta^{R}\right)\right)=0
$$

Equating the real and imaginary parts of Eqs. (21) and (22) give the following identities:

$$
\begin{aligned}
& \alpha^{L} \cdot \alpha^{R}=\beta^{L} \cdot \beta^{R}=1 \\
& \alpha^{L} \cdot \beta^{R}=\alpha^{R} \cdot \beta^{L}=0
\end{aligned}
$$

The contribution to the monodromy matrix from a complex pair of eigenvalues is

$$
\begin{aligned}
\lambda_{+} \mathbf{v}_{+}^{R} \mathbf{v}_{+}^{L}+\lambda_{-} \mathbf{v}_{-}^{R} \mathbf{v}_{-}^{L} & =\frac{1}{2}\left(\alpha^{R}+i \beta^{R}\right)\left(\alpha^{L}-i \beta^{L}\right) e^{i \theta}+\frac{1}{2}\left(\alpha^{R}-i \beta^{R}\right)\left(\alpha^{L}+i \beta^{L}\right) e^{-i \theta} \\
& =\frac{1}{2}\left(\alpha^{R} \alpha^{L}+\beta^{R} \beta^{L}\right)\left(e^{i \theta}+e^{-i \theta}\right)+\frac{1}{2} i\left(\beta^{R} \alpha^{L}-\alpha^{R} \beta^{L}\right)\left(e^{i \theta}-e^{-i \theta}\right) \\
& =\cos \theta\left(\alpha^{R} \alpha^{L}+\beta^{R} \beta^{L}\right)+\sin \theta\left(\alpha^{R} \beta^{L}-\beta^{R} \alpha^{L}\right)
\end{aligned}
$$

\section{Dynamic Coordinates}

We wish to choose coordinates to describe the state which convey dynamical meaning. Instead of carrying over the coordinate basis from the linearization of the Lagrangian or Hamiltonian coordinate frame, we will make use the linear manifolds, surface of section, and energy concepts. The energy component is the only component that cannot be dealt with using the available eigenvectors of the monodromy matrix. The six coordinates necessary to fully quantify the state, which be described in more detail shortly, are

1. component along the unstable manifold, $a_{u}$

2. component along the stable manifold, $a_{s}$

3. pseudo-magnitude of state projected into the center subspace, $\rho$ 
4. pseudo-angle of the state projected into the center subspace, $\gamma$

5. deviation from the surface of section (downtrack deviation), $a_{d}$

6. deviation in energy, $\delta H$

Defining the columns of the matrix $M^{R}$ as the directions along which to take projections, we have

$$
M^{R}=\left[\begin{array}{llllll}
\mathbf{v}_{u}^{R} & \mathbf{v}_{s}^{R} & \alpha^{R} & \beta^{R} & \mathbf{v}_{d}^{R} & \mathbf{v}_{H}^{R}
\end{array}\right]
$$

The columns of $M^{R}$ are assumed to be normalized unit vectors, except for $\alpha^{R}$ and $\beta^{R}$, which are defined as in Eq. (20a). In their respective order, the first five columns of $M^{R}$ are the unstable eigenvector, stable eigenvector, the $\alpha$ and $\beta$ vectors (from the two complex conjugate pair eigenvectors which are assumed to be normalized), and the downtrack eigenvector; $v_{d}^{R}=\frac{J \frac{d H}{d X}}{\left|J \frac{d H}{d X}\right|}$. The last column is the gradient of the energy with respect to the state: $v_{H}^{R}=\frac{d H / d X}{|d H / d X|}$.

Define the dual basis as $M^{L}=\left(M^{R}\right)^{-1}$. Due to our definition of $M^{R}$ (we aren't strictly using only eigenvectors), the rows of $M^{L}$ are no longer left-eigenvectors of the monodromy matrix, however, the fact that they form a dual basis still gives us the useful property that $v_{i}^{L} \cdot v_{j}^{R}=\delta_{i j}$. With this in mind, let us partition $M^{L}$ as

$$
M^{L}=\left[\begin{array}{c}
\mathbf{v}_{u}^{L} \\
\mathbf{v}_{s}^{L} \\
\alpha^{L} \\
\beta^{L} \\
\mathbf{v}_{d}^{L} \\
\mathbf{v}_{H}^{L}
\end{array}\right]
$$

The coordinates of along the unstable, stable, and downtrack manifolds as well as the coordinate along the energy gradient direction are given by simple dot products:

$$
\begin{aligned}
a_{u} & =\mathbf{x} \cdot \mathbf{v}_{u}^{L} \\
a_{s} & =\mathbf{x} \cdot \mathbf{v}_{s}^{L} \\
a_{d} & =\mathbf{x} \cdot \mathbf{v}_{d}^{L} \\
\delta H & =\mathbf{x} \cdot \mathbf{v}_{H}^{L}
\end{aligned}
$$

For the center manifold, we can assume that the state is partitioned into $\mathbf{x}=\cdots+a \alpha^{R}+b \beta^{R}+\cdots$. The coefficients $a$ and $b$ are found by dotting with the left vectors; $a=\mathbf{x} \cdot \alpha^{L}$ and $b=\mathbf{x} \cdot \beta^{L}$. Now if we assume that $a=\rho \cos \gamma$ and $b=\rho \sin \gamma$, we can solve for our center manifold components, $\rho$ and $\gamma$ :

$$
\begin{aligned}
& \rho=\sqrt{a^{2}+b^{2}} \\
& \gamma=\tan ^{-1} \frac{b}{a}
\end{aligned}
$$

It should be noted that $\rho$ and $\gamma$ are not strictly a magnitude and angle since, in general, $\alpha$ and $\beta$ are not orthogonal. Nevertheless, they still provide a useful interpretation of how the state projects into the center manifold. For instance, with no control acting, $\gamma$ will be nearly constant throughout the motion.

\section{Generalized Eigenvectors}

An alternative choice to using the energy gradient for capturing the local state is to use a generalized eigenvector. Let the columns of the matrix $\hat{R}$ be the true and generalized eigenvectors. This method has the advantage that when the dual basis is computed by $\hat{L}=\hat{R}^{-1}$, the rows of $\hat{L}$ are either true left eigenvectors or the generalized left eigenvector. The disadvantage is that to calculate the energy deviation using this generalized left eigenvector, we have to account for the fact that the generalized left eigenvector will generally have components in the energy gradient direction as well as the other directions. Therefore, the calculation of energy deviation is more straightforward using the energy gradient as the remaining direction. Since energy deviation is a desired coordinate to use for this analysis, the energy gradient is a more natural choice. 
As defined in Chen, ${ }^{5}$ the vector $\mathbf{v}$ is a generalized eigenvector of grade $k$ of matrix $A$ associated with eigenvalue $\lambda$ if and only if

$$
\begin{array}{r}
(A-\lambda I)^{k} \mathbf{v}=\mathbf{0} \\
(A-\lambda I)^{k-1} \mathbf{v} \neq \mathbf{0}
\end{array}
$$

In general, the computation proceeds by finding the smallest integer $k$ such that $\operatorname{rank}\left((A-\lambda I)^{k}\right)=n-m$, where $n$ is the dimension of $A$ and $m$ is the algebraic multiplicity of $\lambda$. In our case, the algebraic multiplicity of $\lambda=1$ is two and one true eigenvector is guaranteed to exist, therefore we know we only need to consider $k=2$. To find a solution to Eqs. (25) and (26), we may begin by inserting the eigenvectors of $(\Phi-I)^{2}$ into the matrix $R_{g}$. We then note which columns of $(\Phi-I)^{2} R_{g}$ are the zero vector and which columns of $(\Phi-I) R_{g}$ are not the zero vector. The intersection of these two sets of columns results in one vector, $\mathbf{g}$. Any multiple of this vector may be taken as the generalized eigenvector, $\mathbf{v}_{g}=\alpha \mathbf{g}$, where $\alpha$ is scalar. Once $\mathbf{v}_{g}$ is chosen, the corresponding true eigenvector, $\mathbf{v}$, is then given by

$$
\mathbf{v}=(\Phi-I) \mathbf{v}_{g} .
$$

To keep $\mathbf{v}$ a unit vector like the other eigenvectors, we may choose the generalized eigenvector to be scaled as

$$
\mathbf{v}_{g}= \pm \frac{\mathbf{g}}{\|(\Phi-I) \mathbf{g}\|}
$$

\section{Example: Planar Restricted Two-Body Problem}

The planar restricted two-body problem has two properties that make it useful when studying the eigenstructure of the monodromy matrix. First, periodic orbits are easily obtained analytically. Second, circular orbits have a timeinvariant linearization when the problem is formulated in a rotating frame. This allows for analytical computation of the monodromy matrix by matrix exponentiation. The Hamiltonian for this simple system is ${ }^{6}$

$$
H=\frac{p_{r}^{2}}{2 m}+\frac{p_{\theta}^{2}}{2 m r^{2}}-\frac{\mu m}{r}
$$

where $m$ is the mass of the particle and $\mu$ is the gravitational parameter of the central body. The spatial coordinates are $r$, the distance of the particle from the central body, and $\theta$, the angle the particle makes in the plane with respect to an inertial reference. The momenta coordinates are $p_{r}$, the radial momentum (equal to $m \dot{r}$ ) and $p_{\theta}$, the angular momentum of the particle (equal to $m r^{2} \dot{\theta}$ ). The dynamics for this system formulated as a Hamiltonian system are given by

$$
\dot{\mathbf{X}}=J H_{\mathbf{x}}=\left[\begin{array}{c}
\frac{p_{r}}{m} \\
\frac{p_{\theta}}{m r^{2}} \\
\frac{p_{\theta}}{m r^{3}}-\frac{\mu m}{r^{2}} \\
0
\end{array}\right]
$$

where $\mathbf{X}=\left[\begin{array}{llll}r & \theta & p_{r} & p_{\theta}\end{array}\right]^{\mathrm{T}}, J=\left[\begin{array}{cc}0_{2 \times 2} & I_{2} \\ -I_{2} & 0_{2 \times 2}\end{array}\right]$, and $H_{\mathbf{x}}=\frac{\partial H}{\partial \mathbf{x}}$. Taking $m=\mu=1$ for this example, a valid solution to the nonlinear equations of motion is a circular orbit at a radius $r=1$,

$$
\mathbf{X}(t)=\left[\begin{array}{llll}
1 & t & 0 & 1
\end{array}\right]^{\mathrm{T}}
$$

The linearization about this time-varying solution is time-invariant because the $\theta$ coordinate does not actually appear in the equations of motion:

$$
\delta \dot{\mathbf{X}}=\left[\begin{array}{c}
\delta \dot{r} \\
\delta \dot{\theta} \\
\delta \dot{p}_{r} \\
\delta \dot{p}_{\theta}
\end{array}\right]=\left[\begin{array}{cccc}
0 & 0 & 1 & 0 \\
-2 & 0 & 0 & 1 \\
-1 & 0 & 0 & 2 \\
0 & 0 & 0 & 0
\end{array}\right]\left[\begin{array}{c}
\delta r \\
\delta \theta \\
\delta p_{r} \\
\delta p_{\theta}
\end{array}\right]=A \delta \mathbf{X}
$$


The state transition matrix may be calculated using a matrix exponential:

$$
\Phi(t)=e^{A t}=\left[\begin{array}{cccc}
\cos (t) & 0 & \sin (t) & 2(1-\cos (t)) \\
-2 \sin (t) & 1 & 2(\cos (t)-1) & 4 \sin (t)-3 t \\
-\sin (t) & 0 & \cos (t) & 2 \sin (t) \\
0 & 0 & 0 & 1
\end{array}\right]
$$

These linear equations could also be obtained from the Clohessy-Wiltshire equations ${ }^{7}$ once converted from their Lagrangian frame to this Hamiltonian frame. The eigenvalues of the $A$ matrix are $\pm i$ and 0 (with multiplicity two). Therefore, the eigenvalues of $\Phi(t)$ are $e^{ \pm i t}$ and 1 (with multiplicity two). The eigenvectors of both $A$ and $\Phi(t)$ are $\left[\begin{array}{llll}1 & \pm 2 i & \pm i & 0\end{array}\right]^{\mathrm{T}}$ and $\left[\begin{array}{llll}0 & 1 & 0 & 0\end{array}\right]^{\mathrm{T}}$ which do not span the phase space. The eigenvector associated with the unity eigenvalue of $\Phi$ could be predicted from the dynamics:

$$
J H_{\mathbf{X}}=\left[\begin{array}{llll}
0 & 1 & 0 & 0
\end{array}\right]^{\mathrm{T}}
$$

Additionally, the direction not spanned by the eigenvectors is the direction corresponding to the gradient of the Hamiltonian:

$$
H_{\mathbf{X}}=\left[\begin{array}{llll}
0 & 0 & 0 & 1
\end{array}\right]^{\mathrm{T}}
$$

In this case, the generalized eigenvector may be chosen as

$$
\mathbf{v}_{g}=\left[\begin{array}{llll}
0 & 0 & 0 & -1 /(6 \pi)
\end{array}\right]^{\mathrm{T}}
$$

which results in the eigenvector

$$
\mathbf{v}=\left[\begin{array}{llll}
0 & 1 & 0 & 0
\end{array}\right]^{\mathrm{T}}
$$

\section{Application to Hill Three-Body Problem}

In this section we will study two cases of spacecraft control in the Hill three-body problem (H3BP) using continuous thrust. In the first case, we limit ourselves to the planar motion of a spacecraft in the vicinity of one of the relative equilibrium points, and in the second, we study a spacecraft perturbed from a nominal halo orbit.

The equations of motion for a spacecraft's position in the H3BP, expressed in the standard Lagrangian frame, are ${ }^{8}$

$$
\begin{aligned}
\ddot{x}-2 \omega \dot{y} & =-\frac{\mu}{r^{3}} x+3 \omega^{2} x+a_{x} \\
\ddot{y}+2 \omega \dot{x} & =-\frac{\mu}{r^{3}} y+a_{y} \\
\ddot{z} & =-\frac{\mu}{r^{3}} y-\omega^{2} z+a_{z},
\end{aligned}
$$

where $x, y$, and $z$ are the positions of the spacecraft in the rotating frame relative to the secondary body, $a_{x}, a_{y}$, and $a_{z}$ are the spacecraft control accelerations, $\omega$ is the angular velocity of the secondary body about the primary, $\mu=G M, M$ is the mass of the secondary body, and $r$ is the radius $\left(r=\sqrt{x^{2}+y^{2}+z^{2}}\right)$. These equations may be nondimensionalized using the length scale $l=\left(\mu / \omega^{2}\right)^{1 / 3}$ and time scale $\tau=1 / \omega$. For the Earth-Sun system, $\mu=3.986 \times 10^{5} \mathrm{~km}^{3} / \mathrm{s}^{2}, \omega=$ $1.991 \times 10^{-7} \mathrm{rad} / \mathrm{s}, l=2.159 \times 10^{6} \mathrm{~km}$, and $\tau=5.023 \times 10^{6} \mathrm{~s}$. In this paper, we will study the nondimensionalized system obtained by setting $\mu=\omega=1$. When expressed in the Hamiltonian frame, we have the following first order equations of motion:

$$
\begin{aligned}
\dot{x} & =p_{x}+y \\
\dot{y} & =p_{y}-x \\
\dot{z} & =p_{z} \\
\dot{p}_{x} & =p_{y}-\frac{x}{r^{3}}+2 x \\
\dot{p}_{y} & =-p_{x}-y-\frac{y}{r^{3}} \\
\dot{p}_{z} & =-\frac{z}{r^{3}}-z
\end{aligned}
$$




\section{A. Equilibrium Point of H3BP}

This Hamiltonian system has two equilibrium points using no control at $x= \pm 3^{-1 / 3}, p_{y}=x$, and $y=z=p_{x}=p_{z}=0$. Linearizing about either of these points and defining the perturbed state $\delta \mathbf{x}=\left[\begin{array}{llll}\delta x & \delta y & \delta p_{x} & \delta p_{y}\end{array}\right]^{\mathrm{T}}$ yields the linear system

$$
\delta \dot{\mathbf{x}}=\left[\begin{array}{cccc}
0 & 1 & 1 & 0 \\
-1 & 0 & 0 & 1 \\
8 & 0 & 0 & 1 \\
0 & -4 & -1 & 0
\end{array}\right] \delta \mathbf{x}+\left[\begin{array}{ll}
0 & 0 \\
0 & 0 \\
1 & 0 \\
0 & 1
\end{array}\right]\left[\begin{array}{l}
a_{x} \\
a_{y}
\end{array}\right]
$$

This system has an unstable mode, a stable mode, and an oscillatory mode, associated with the eigenvalues $+\sqrt{1+2 \sqrt{7}} \approx$ $2.5,-\sqrt{1+2 \sqrt{7}} \approx-2.5$, and $\pm j \sqrt{2 \sqrt{7}-1} \approx \pm 2.1 j$, respectively.

We apply these coordinates to both controlled and uncontrolled trajectories near the equilibrium point. For the controlled system, the control is the optimal control which drives the state from a perturbed position back to the equilibrium point (the origin) in a finite time while minimizing the "energy" used;

$$
J=\frac{1}{2} \int_{t_{0}}^{t_{f}}\left(a_{x}^{2}+a_{y}^{2}\right) d t
$$

In this example, we use $t_{0}=0$ and $t_{f}=4$.

Figure 1 show the coordinates for an uncontrolled system. Note the each component behaves as expected - the unstable component grows exponentially, the stable component converges exponentially, the pseudo-angle and pseudomagnitude are nearly constant.

The optimally controlled system's coordinates are shown in Figure 2. As one would expect, the control acts to quickly diminish the state along the unstable direction. The components along the stable, unstable, and pseudomagnitude directions are all 0 at the final time.

\section{B. Halo Orbits in H3BP}

From the two oscillatory modes mentioned in the previous section, we see that near the equilibrium point, the linearized system is capable of producing planar periodic orbits. These orbits can also be found in the full nonlinear dynamics. As the amplitude of these periodic orbits is increased, the eigenvalues of the monodromy matrix bifurcate and a new family of periodic orbits is produced. This new family is called the family of "halo orbits", which are no longer in the plane and cannot be predicted using the equilibrium point linearization. The halo orbit used in this example, shown in Figure 3, has initial conditions approximately $x_{0}=0.769, z_{0}=0.18698, p_{y_{0}}=0.853444, y_{0}=p_{x_{0}}=p_{z_{0}}=0$, and period $T \approx 3.07$.

As in the planar case, we shown the behavior of an uncontrolled and an optimally controlled system. The optimal control is still the "energy", this time with three inputs;

$$
J=\frac{1}{2} \int_{t_{0}}^{t_{f}}\left(a_{x}^{2}+a_{y}^{2}+a_{z}^{2}\right) d t .
$$

For these cases, we use $t_{0}=0$ and $t_{f}=T$.

Figure 4 show the coordinates for an uncontrolled system. Note the simplistic behavior of the coordinates; the stable and unstable coordinates both decay and grow exponentially over an orbit, the pseudo-magnitude and downtrack components are nearly periodic, the pseudo-angle is nearly constant, and energy deviation is extremely small. The optimally controlled system's coordinates are shown in Figure 5.

\section{Conclusions}

We propose a method to analyze motion near halo orbits. The Hamiltonian nature of the system allows for special analysis of the eigenstructure of the monodromy matrix, particularly the direction not spanned by its eigenvectors. In addition, special attention is given to the components of the center subspace in order to give more physical insight. We show the eigenstructure of a simple circular orbit in the two body problem, and apply the coordinates to motion near both an equilibrium point and a halo in the Hill three-body problem. 

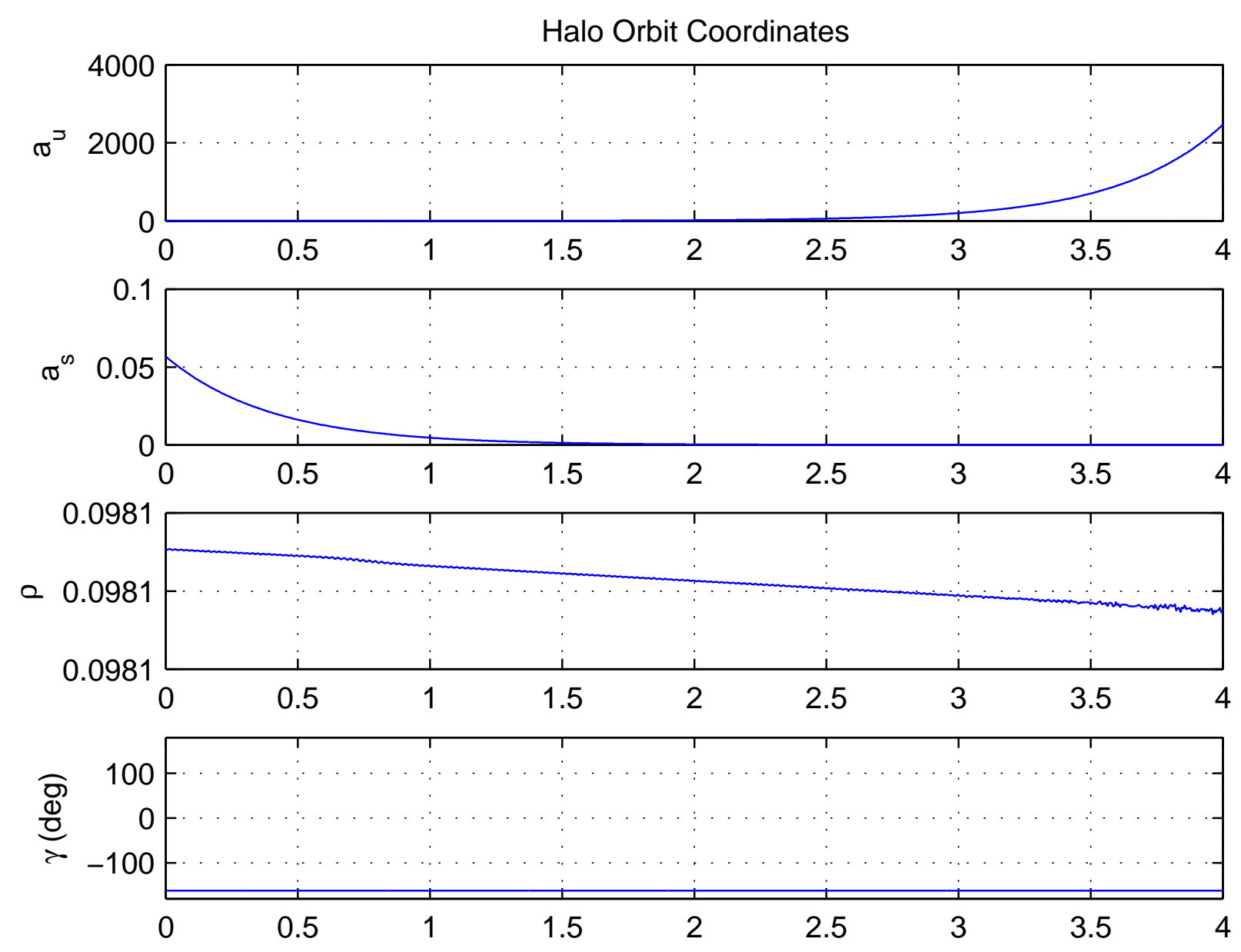

Figure 1. Uncontrolled trajectory near equilibrium point

\section{References}

${ }^{1}$ D.J. Scheeres, B.G. Williams, and J.K. Miller. Evaluation of the dynamic environment of an asteroid: Applications to 433 eros. Journal of Guidance, Control, and Dynamics, 23(3), May-June 2000.

${ }^{2}$ C. Marchal. The Three Body Problem. Elsevier, 1989.

${ }^{3}$ Ernst Hairer, Christian Lubich, and Gerhard Wanner. Geometric Numerical Integration: Structure Preserving Algorithms for Ordinary Differential Equations. Springer, 2nd edition, 2004.

${ }^{4}$ Kathleen Howell. Three-dimensional, periodic, 'halo' orbits. Celestial Mechanics and Dynamical Astronomy, 32(1):53-71, January 1984.

${ }^{5}$ Chi-Tsong Chen. Linear System Theory and Design. The Oxford Series in Electrical and Computer Engineering. Oxford University Press, 1984.

${ }^{6}$ Donald T. Greenwood. Classical Dynamics. Dover Publications, Inc., 1977.

${ }^{7}$ John E. Prussing and Bruce A. Conway. Orbital Mechanics. Oxford University Press, 1993.

${ }^{8}$ C. A. Renault and D. J. Scheeres. Statistical analysis of control maneuvers in unstable orbital environments. Journal of Guidance, Control, and Dynamics, 26(5), September-October 2003. 

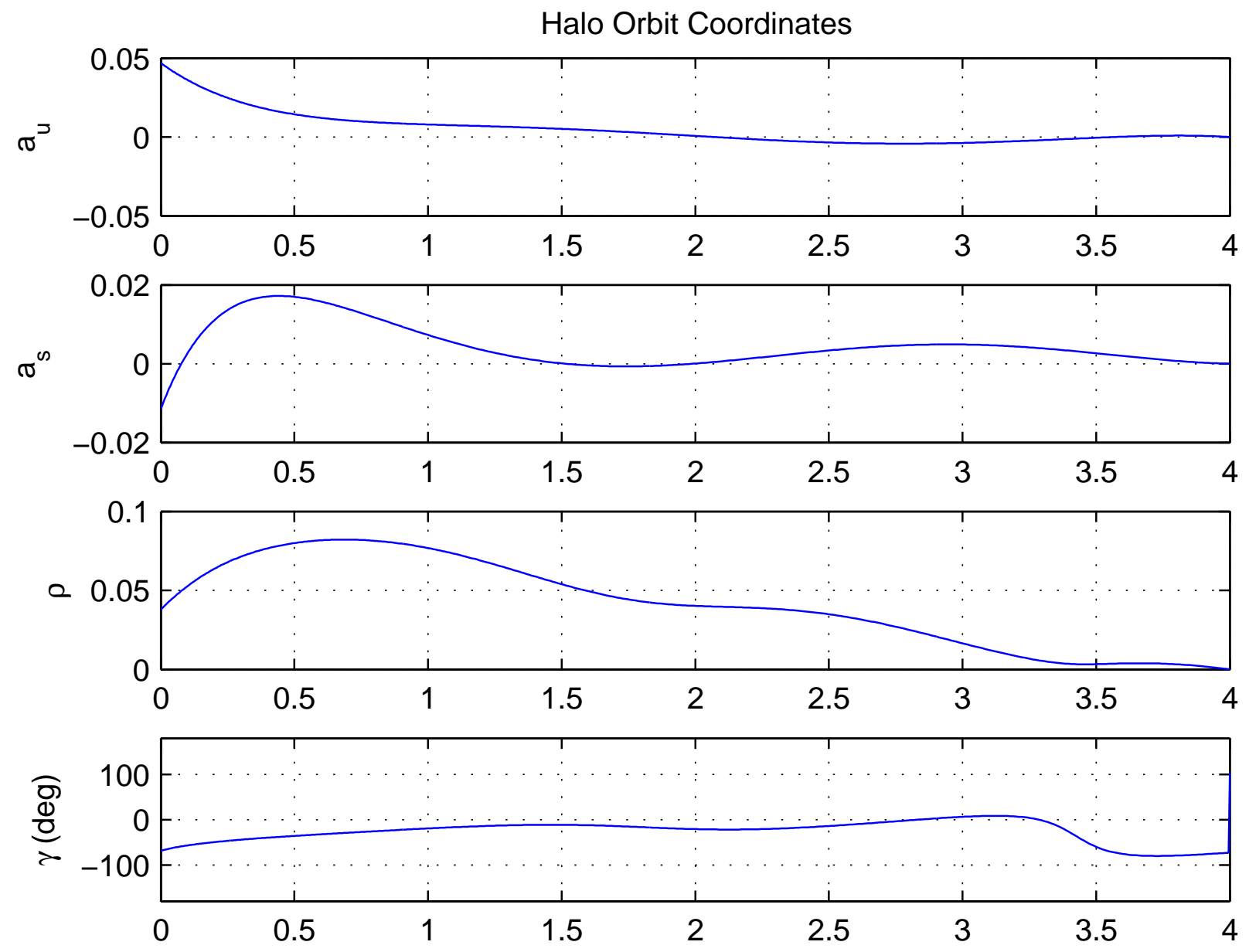

Figure 2. Controlled trajectory near equilibrium point 

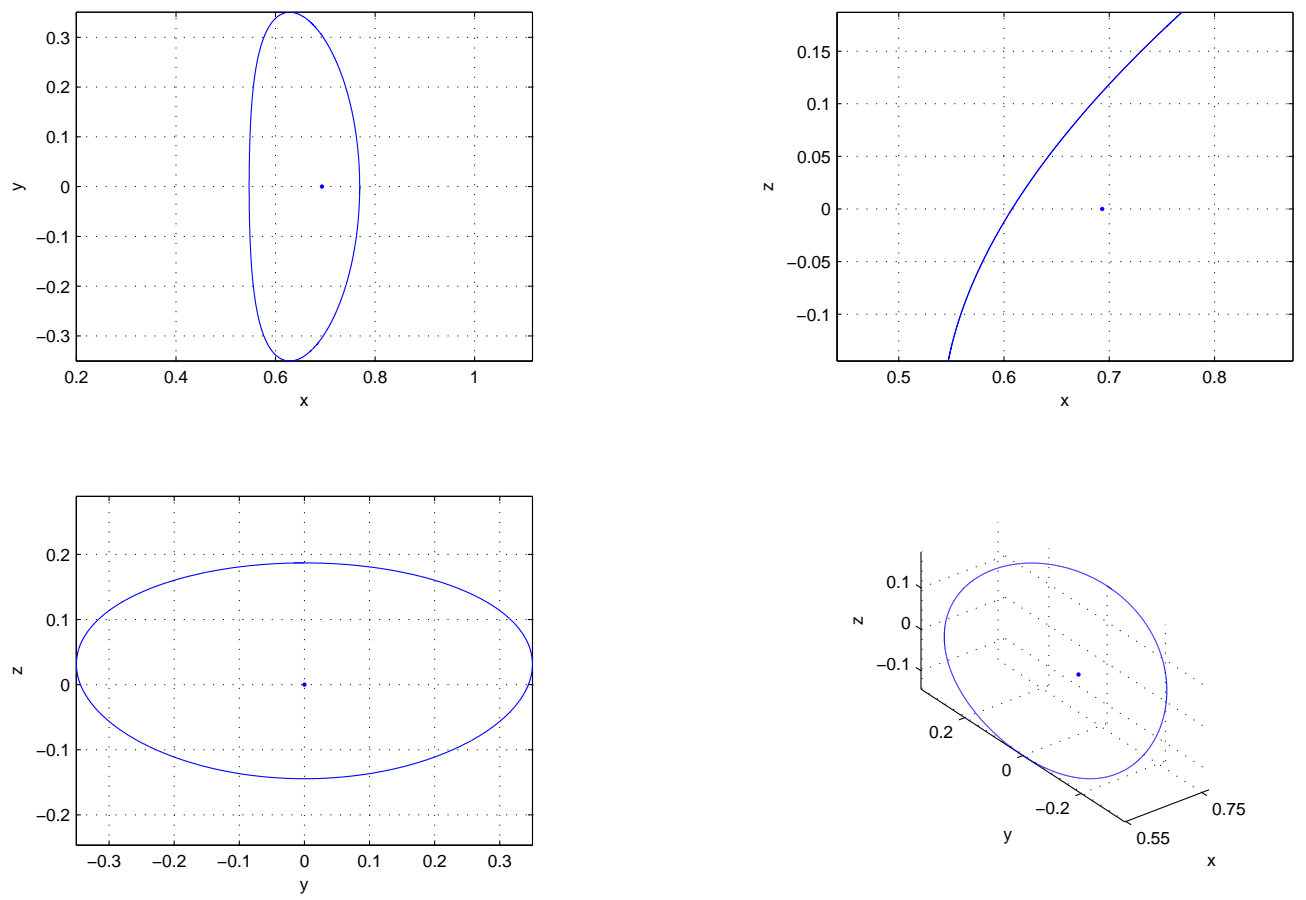

Figure 3. Example halo orbit. The equilibrium point is denoted by a dot. 

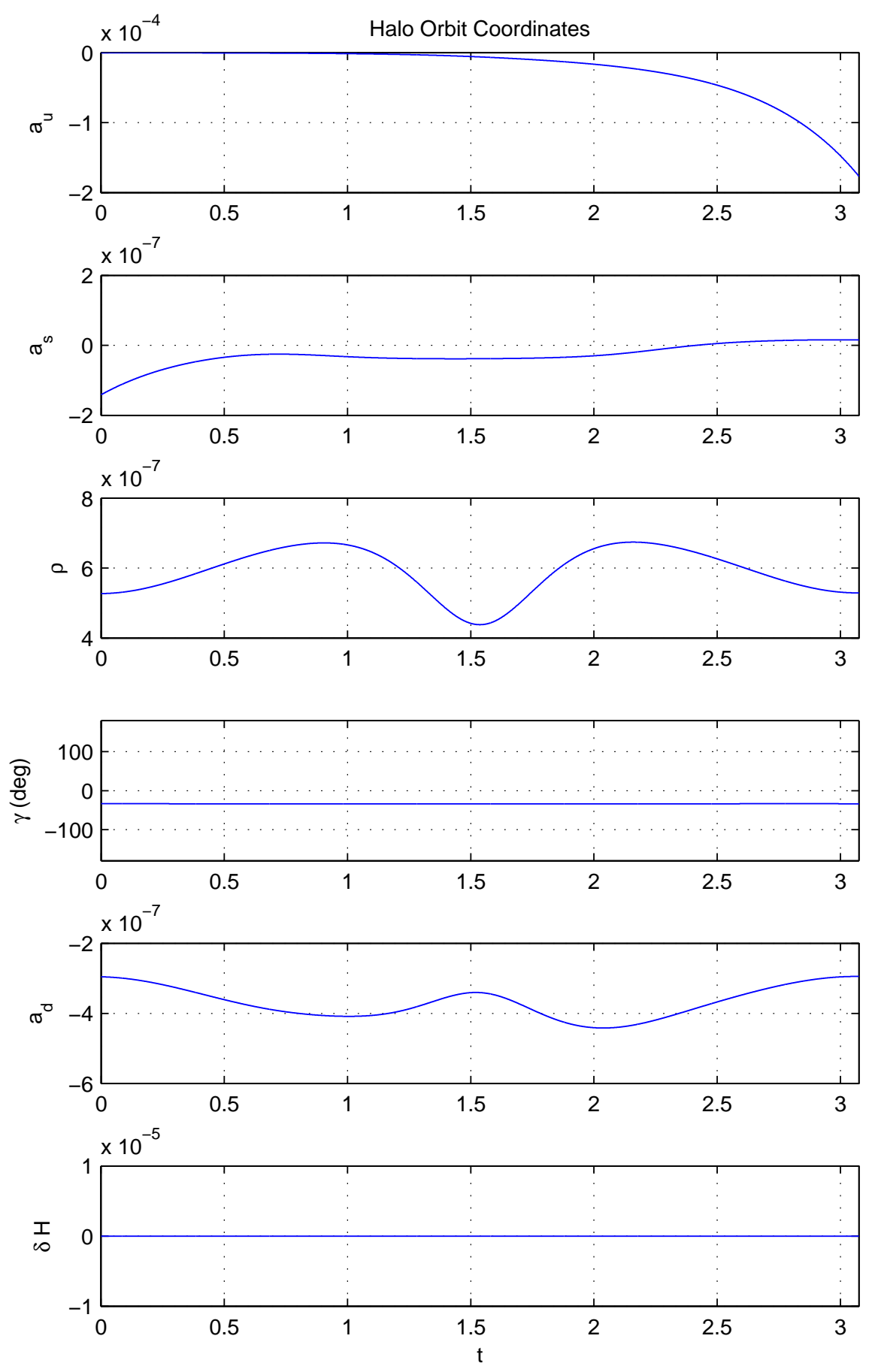

Figure 4. Uncontrolled halo system 

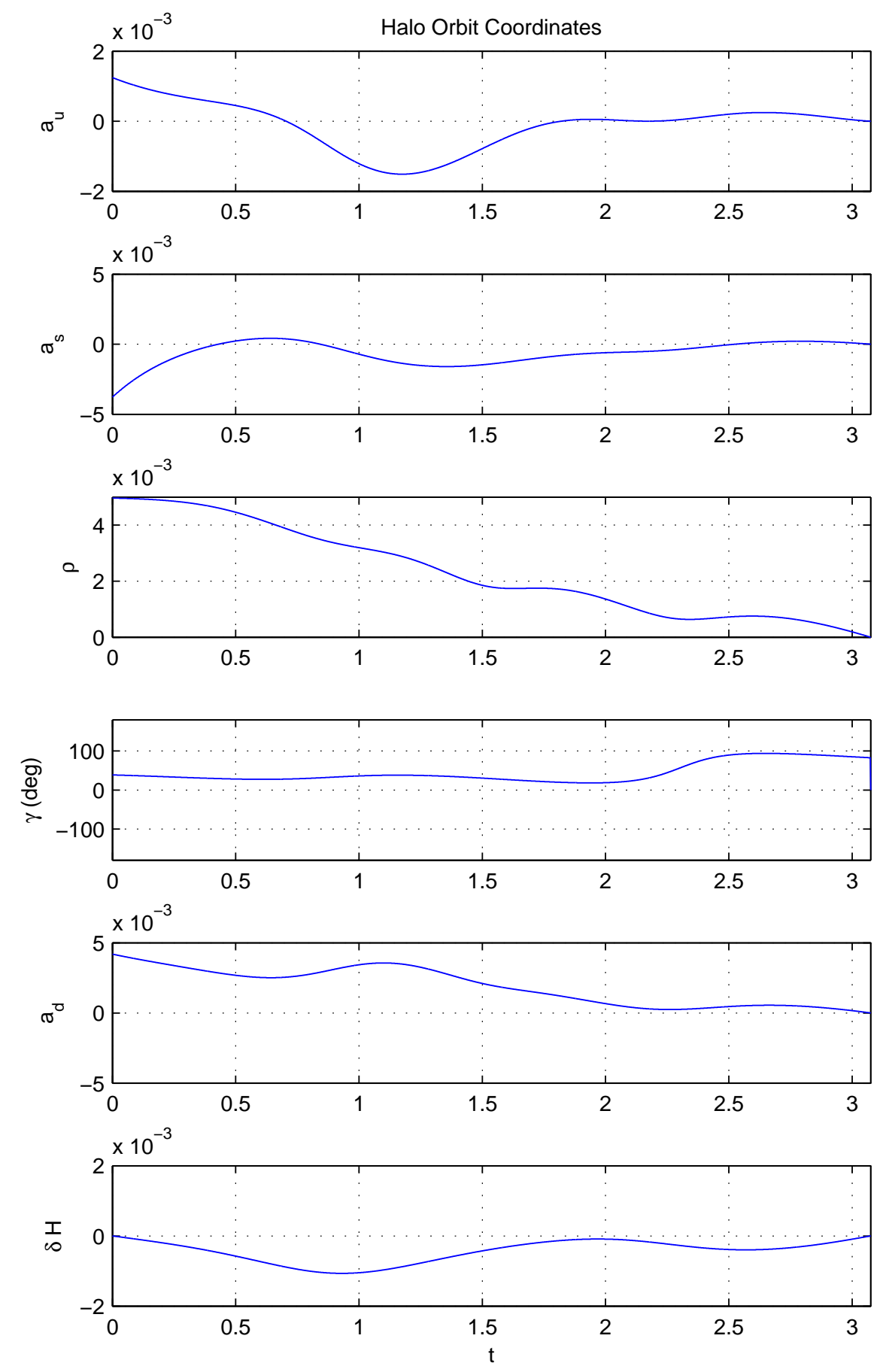

Figure 5. Controlled halo system

16 of 16 